\title{
Humidity sensitivity investigation of reduced graphene oxide by impedance spectroscopy
}

\begin{abstract}
In this chapter we report about graphene oxide (GO) and reduced GO (rGO) humidity sensitivity. The GO was reduced by thermal annealing to form $\mathrm{rGO}$, which has increased its conductivity. The effects of the reduction temperature were investigated for both the electrical and humidity sensitivity properties. The electrical properties were studied by electrochemical impedance spectroscopy, which is in particular of high importance to reveal the conduction mechanism of humidity sensitivity. In addition, the morphology and optical properties of rGO films were investigated by atomic force microscopy and UV-Vis-NIR spectroscopy. The reduction temperature plays an important rule for the rGO humidity sensitivity behavior not only by change in film conductivity but also by the transport mechanism.
\end{abstract}

Keywords: graphene oxide, reduced graphene oxide, humidity sensor, impedance modeling

\section{Introduction}

Graphene and graphene oxide (GO) as carbon-based material with their well-known, outstanding properties have attracted a lot of attention in diverse fields, such as electronic devices like transistors and diodes [1], energy storage [2] and sensors [3, 4]. GO is considered as a very promising material due to its water dispersibility enabling solution processing [5], as well as large-scale production and easy processing. GO was used as humidity sensor either without further processing or by introducing it into a polymer matrix as well as by altering it using chemical functional groups by reduction to obtain reduced GO (rGO) [6, 7]. Reduction of GO can be carried out by several techniques, e. g., thermal, chemical or photonic reductions, by which some of the oxygen containing functional groups are eventually removed partially [8]. The importance of reduction is not only that it converts GO from a semi-insulating to a conductive material but also that it is controllable and tunable. This has a big influence on the GO and rGO electrical and sensing properties where they will be functions of type and degree of the reductions.

GO as a promising material for humidity sensors as well as other types of sensors still needs to be characterized better to explore its tunability in different aspects. The

Ammar Al-Hamry, Renato Veiga de Torres, Christian Müller, Olfa Kanoun, Chair for Measurements and Sensor Technology, Chemnitz University of Technology, Reichenhainer Strasse 70, 09126 Chemnitz, Germany, e-mail: ammar.al-hamry@s2011.tu-chemnitz.de 
ability to lose their oxygen groups by reduction and to show different surface, conductivity and sensitivity properties is of high interest. In this chapter, we investigate the effect of the reduction temperature on the humidity sensitivity of GO films. The measurements were carried out by taking the impedance of rGO films.

\section{Experimental procedures}

GO-coated thin films were prepared by spin coating. The reduction process was carried out by thermal annealing at different temperatures to produce rGO. Electrical contacts were made by silver paste. The humidity measurements were taken under certain relative humidity $(\mathrm{RH})$ conditions by means of saturated slat solution at RH values of $0 \%$, $11.3 \%, 32.5 \%, 52.3 \%, 75.2 \%, 84.3 \%$ and $97.3 \%$ and at room temperature $\left(23^{\circ} \mathrm{C}\right)$. Electrical impedance of the films was measured by the Agilent impedance analyzer 4294A.

\section{Results and discussion}

In Fig. 1, the impedance relation to the humidity of rGO films reduced at different temperatures is shown. The change in impedance by thermal heating is one order of magnitude by increasing heat from $160^{\circ} \mathrm{C}$ to $250^{\circ} \mathrm{C}$, where GO itself is semi-insulating, having a resistance of hundreds of MOhms. The sensitivity behavior shows dependency on the reduction temperature. At low humidity, the impedance increases for all rGO films. At high humidity, the impedance tends to decrease and shows a negative slope. However, for the films reduced at higher temperature, the impedance shows a positive slope. The relation is quite linear at lower humidity values.

In the Nyquist plot, Fig. 2 (a) and (b), a semicircle fits the parallel resistance and capacitance, which represent the intrinsic impedance of the film. The behavior at low

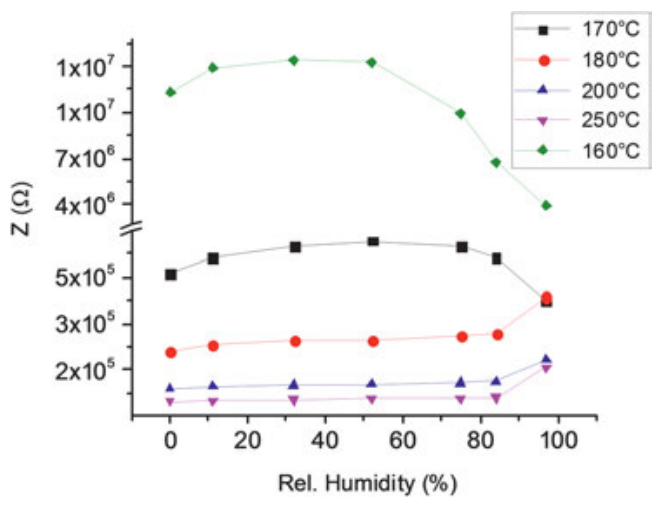

Fig. 1: Relation of impedance of $\mathrm{rGO}$ films with $\mathrm{RH}$ at $100 \mathrm{~Hz}$. 
humidity range (0-52\%) shows an increase of the semicircle due to absorbed water molecules. This causes to charge transfer between water molecules and rGO, which reduces the charge carriers of the film [7]. This is applied for all the films. At high humidity (75-97\%), in the Nyquist plot of Fig. 2 (b), the semicircle with small tail shows a constant phase element (CPE) behavior. The equivalent circuit consists of two CPEs and a resistance that represents the interaction of water and contact and intrinsic resistance.
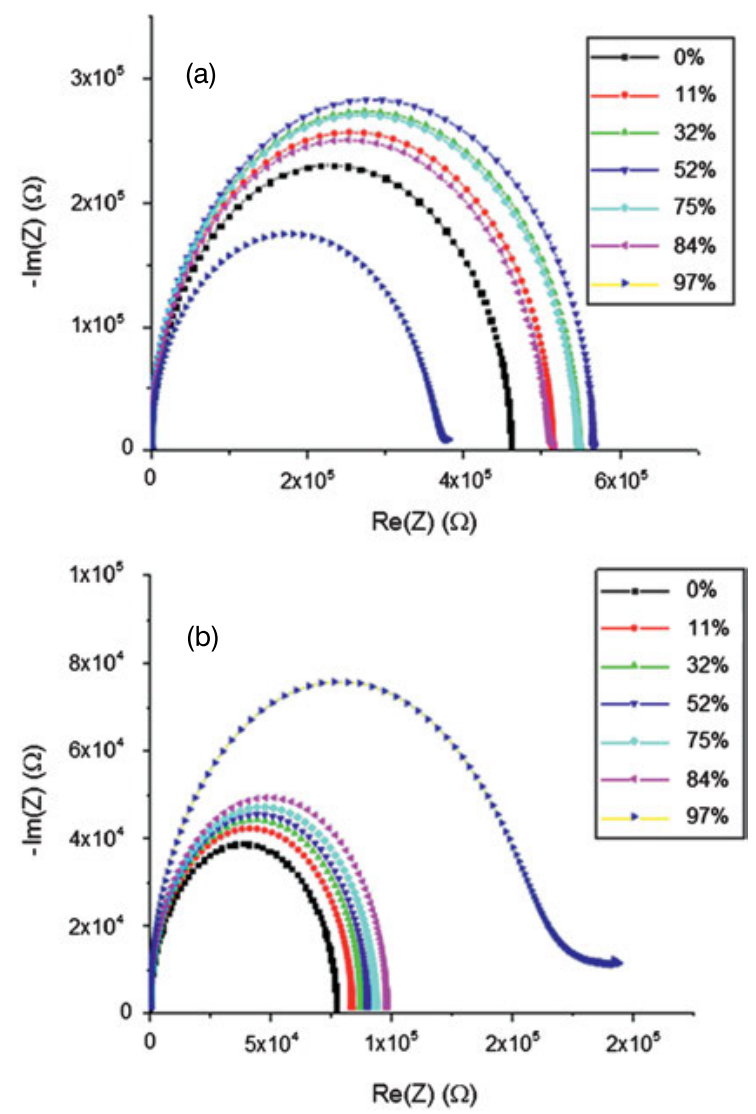

Fig. 2: Nyquist plot for (a) rGo reduced at $170^{\circ} \mathrm{C}$ and (b) $\mathrm{rGO}$ reduced at $200^{\circ} \mathrm{C}$ under different $\mathrm{RH}$ conditions.

For rGO reduced at higher temperatures, by increase in the number of water molecules, the charge transfer is dominant and causes a further increase of the impedance. For low-temperature reduced films, the formation of water thin films has the role of decreasing the impedance due to proton hopping [9]. In addition, by increasing the reduction temperature, rGO films become more hydrophobic, which influences the formation of a continuous water film. Thus the impedance only further increases at higher values of humidity. 


\section{Summary and conclusion}

GO coated and reduced by temperature leads to tuning not only the conductivity but also the film sensitivity to humidity. The impedance can be reduced from hundreds of MOhms to tens of $\mathrm{kOhms}$ by heating the films to $250^{\circ} \mathrm{C}$. The reduction by temperature results in different humidity sensitivity behaviors. Tuning the reduction temperature provides useful selectivity of the humidity sensitivity. Where at lower temperature is more sensitive at higher humidities, at high reduction temperatures, the sensitivity to humidity becomes less due to the increase of the hydrophobicity of the rGO films. However, it improves the linearity.

The realization of a humidity sensor or sensor matrix that covers the full range of humidity is also foreseen, e. g., by combining two films with two different annealing temperatures and thus different transport mechanisms at low and high temperature. The electrical impedance was obtained by impedance spectroscopy, which reveals the conduction mechanism. However, further tests and study of the equivalent circuit models will be a next step, which will provide an in-depth understanding of the different behaviors in humidity sensitivity of rGO.

\section{Bibliography}

[1] J. Basu, J. K. Basu, and T. K. Bhattacharyya, "The evolution of graphene-based electronic devices", International Journal of Smart and Nano Materials, pp. 201-223, 2010.

[2] R. Mukherjee, A. V. Thomas, D. Datta, E. Singh, O. Eksik et al., "Defect-induced plating of lithium metal within porous graphene networks", Nature Communications, 2014.

[3] Z. Bo, X. Shuai, S. Mao, H. Yang, J. Qian, J. Chen et al., “Green preparation of reduced graphene oxide for sensing and energy storage applications”, Scientific Reports, 2014.

[4] O. Kanoun, C. Müller, A. Benchirouf, A. Sanli, T. Dinh, A. Al-Hamry et al., "Flexible carbon nanotube films for high performance strain sensors”, Sensors, 2014.

[5] G. Eda and M. Chhowalla, "Chemically derived graphene oxide: towards large-area thin-film electronics and optoelectronics”, Advanced Materials, 2010.

[6] S. Borini, R. White, Di. Wei, M. Astley, S. Haque, E. Spigone et al., "Ultrafast graphene oxide humidity sensors”, ACS Nano, vol. 7, 2013.

[7] D. Zhang, J. Tong, and B. Xia, "Humidity-sensing properties of chemically reduced graphene oxide/polymer nanocomposite film sensor based on layer-by-layer nano self-assembly", Sensors and Actuators B, Chemical, vol. 197, 2014.

[8] A. Al-Hamry, H. Kang, E. Sowade, V. Dzhagan, R. Rodriguez, C. Müller, D. Zahn, R. Baumann, and $\mathrm{O}$. Kanoun, "Tuning the reduction and conductivity of solution-processed graphene oxide by intense pulsed light", Journal of Carbon, vol. 102, 2016.

[9] D. Zhang, J. Tong, B. Xia, and Q. Xue, "Ultrahigh performance humidity sensor based on layer-by-layer self-assembly of graphene oxide/polyelectrolyte nanocomposite film", Sensors and Actuators B, Chemical, vol. 203, 2014. 